\title{
Respuesta inmune humoral y celular a la vacuna Brucella abortus cepa RB51 en vaquillas en pastoreo suplementadas con selenio y $\alpha$-tocoferol ${ }^{\#}$
}

\author{
Humoral and cellular immune response to Brucella abortus strain RB51 vaccine \\ in grazing heifers supplemented with selenium and $\alpha$-tocopherol \\ V Leyán ${ }^{a^{*}}$, R Chihuailaf $^{\mathrm{b}}, \mathrm{M}_{\text {Ortega }}{ }^{\mathrm{a}}$ \\ anstituto de Inmunología, Universidad Austral de Chile, Valdivia, Chile. \\ ${ }^{\text {b} E s c u e l a ~ d e ~ M e d i c i n a ~ V e t e r i n a r i a, ~ U n i v e r s i d a d ~ C a t o ́ l i c a ~ d e ~ T e m u c o, ~ T e m u c o, ~ C h i l e . ~}$
}

\begin{abstract}
SUMMARY
In order to evaluate the effect of selenium and $\alpha$-tocopherol supplementation on the immune response to Brucella abortus strain RB51, four groups of six grazing heifers were used. Three months before the start of the trial, the groups Se-T and Se were supplemented in single dose with barium selenate ( $1 \mathrm{mg}$ selenium/kg body weigth) and groups Se-T and T were supplemented with $500 \mathrm{IU} \alpha$-tocopherol/100 $\mathrm{kg}$ each two months. Group C was maintained without supplementation. Once established differences in metabolic balance groups, the trial began with the RB51 vaccine administration. Blood samples with heparin and without additive were obtained from each cow for the determining blood activity of glutathione peroxidase (GPx) and plasma concentration of cholesterol and $\alpha$-tocopherol. The humoral immune response was evaluated by ELISA and the cellular immune response by an intradermal test antigen of $B$. abortus. Differences between groups were evaluated by tests repeated measures ANOVA, paired samples t and KruskalWallis. It was considered significant when the $\mathrm{P}$ value was less than 0.05 . Supplementation with selenium and $\alpha$-tocopherol established differences between groups $(\mathrm{P}<0.05)$. The humoral immune response and cellular response to RB51 vaccine was lower in group $\mathrm{T}(\mathrm{P}<0.05)$. It is possible to conclude that humoral and cellular immune response to the vaccine RB51 can be negatively modulated by plasma $\alpha$-tocopherol concentration at the immunization.
\end{abstract}

Key words: immune response, selenium, $\alpha$-tocopherol, Brucella abortus.

\section{RESUMEN}

Con el objetivo de evaluar el efecto de una suplementación con selenio y $\alpha$-tocoferol sobre la respuesta inmune a la vacuna Brucella abortus cepa RB51, se emplearon cuatro grupos de seis vaquillas en pastoreo. Tres meses previo al inicio del ensayo, los grupos Se-T y Se fueron suplementados en dosis única con selenato de bario ( $1 \mathrm{mg}$ selenio/kg peso vivo) y los grupos Se-T y T con $500 \mathrm{UI}$ de $\alpha$-tocoferol/100 $\mathrm{kg}$ cada dos meses. El grupo C fue mantenido sin suplementación. Una vez establecidas las diferencias en el balance metabólico en los grupos, el ensayo se inició con la administración de la vacuna RB51. De cada vaquilla se obtuvieron muestras de sangre con heparina y sin aditivo con la finalidad de determinar la actividad sanguínea de glutatión peroxidasa (GPx) y las concentraciones plasmáticas de colesterol y $\alpha$-tocoferol. La respuesta inmune humoral se evaluó mediante ELISA y la respuesta inmune celular mediante una prueba de intradermorreacción a antígenos de $B$. abortus. Las diferencias entre grupos se evaluaron mediante las pruebas ANDEVA de medidas repetidas, $\mathrm{t}$ de muestras pareadas y Kruskal-Wallis. Se consideró significativo cuando el valor de $\mathrm{P}$ fue menor a 0,05 . La suplementación con selenio y $\alpha$-tocoferol estableció diferencias entre grupos $(\mathrm{P}<0,05)$. La respuesta inmune humoral y la respuesta celular a la vacuna RB51 fue menor en el grupo T $(\mathrm{P}<0,05)$. Es posible concluir que la respuesta inmune celular y humoral puede ser modulada negativamente por la concentración plasmática de $\alpha$-tocoferol al momento de la inmunización.

Palabras claves: respuesta inmune, selenio, $\alpha$-tocoferol, Brucella abortus.

\section{INTRODUCCIÓN}

La enzima glutatión peroxidasa (GPx) se ha utilizado para estimar el estatus de selenio en los bovinos y es aceptado que un balance adecuado de selenio corresponde a una actividad de GPx superior a $130 \mathrm{U} / \mathrm{g}$ de hemoglobina (Hb) (Ceballos y Wittwer 1996). Un inadecuado balance de selenio puede provocar una disminución de la capacidad

\footnotetext{
Aceptado: 27.08.2014.

\# Financiado parcialmente por Proyectos DID: S-2005-31; S-2006-59 y D-2004-10

* vleyan@uach.cl
}

antioxidante del organismo y conducir a la presentación de estrés oxidativo (Chihuailaf y col 2002). El estrés oxidativo generado por la carencia de selenio en rumiantes se ha asociado con alteraciones musculares, reproductivas, tumorales, del crecimiento, de la función tiroidea y también del sistema inmune (López-Alonso y col 1997). Este último aspecto, ampliamente revisado por Finch y Turner (1996), muestra resultados muy dispares en distintos ensayos de suplementación con selenio y $\alpha$-tocoferol, no obstante lo anterior existe evidencia acerca de la estrecha asociación entre el selenio y el $\alpha$-tocoferol como un modulador de la respuesta inmune (Baldi 2005). En los últimos años se ha evidenciado que un bajo aporte nutricional de selenio en vacas de lechería no afecta significativamente la respuesta 
inmune humoral y celular generada por la vacuna de Brucella abortus RB51 (Leyán y col 2005). Sin embargo, los mismos autores (Leyán y col 2006) también demostraron que la suplementación con selenio en vaquillas con un adecuado balance metabólico de este mineral provoca una disminución significativa en la respuesta inmune celular. Este efecto paradójico sugiere que la respuesta a la suplementación con selenio por el sistema inmune es dependiente del balance de selenio del animal. La dificultad en la interpretación del estatus de selenio asociado a una respuesta orgánica también puede evidenciarse en otros aspectos. En este sentido, recientemente un estudio reveló que un inadecuado balance de selenio no necesariamente se refleja en una baja producción o calidad sanitaria de la leche (Ceballos y col 2013).

En consideración a los antecedentes que apoyan la hipótesis de que el balance nutricional de micronutrientes modula la respuesta inmune, se planteó como objetivo evaluar el efecto del selenio y el $\alpha$-tocoferol sobre la respuesta inmune humoral y celular generada por la inmunización con la vacuna de Brucella abortus RB51 en vaquillas.

\section{MATERIAL Y MÉTODOS}

\section{ANIMALES Y GRUPOS EXPERIMENTALES}

Se utilizaron 24 vaquillas Frisón Negro preencaste, entre 15 y 18 meses de edad, y de $253 \pm 25 \mathrm{~kg}$ de peso vivo pertenecientes a un rebaño comercial libre de tuberculosis, leucosis y brucelosis localizado en la comuna de Los Lagos, Chile (39 $52^{\circ}$ S, $72^{\circ} 34^{\prime}$ O), y con antecedentes de praderas con un bajo contenido de selenio. Los animales se distribuyeron al azar en cuatro grupos de seis vaquillas cada uno.

Tres meses previos al inicio del estudio se establecieron los grupos experimentales: un grupo fue suplementado con selenio (Se), un grupo suplementado solo con $\alpha$-tocoferol (T), un grupo suplementado con selenio y $\alpha$-tocoferol (Se-T) y un grupo control mantenido sin suplementación (C). Las vaquillas de los grupos Se-T y Se fueron suplementados con $1 \mathrm{mg}$ selenio/ $\mathrm{kg}$ vía s.c. en dosis única con selenato de bario ${ }^{1}$. Los grupos Se-T y T fueron suplementados con 500 UI de $\alpha$-tocoferol ${ }^{2} / 100 \mathrm{~kg}$ vía s.c. y luego cada 60 días en dos oportunidades.

Como parte del programa de manejo sanitario del predio, todos los animales son vacunados con la vacuna de $B$. abortus cepa RB51 entre los 6 y 8 meses de edad. Este ensayo se inició con la revacunación de los animales experimentales con la vacuna $B$. abortus (día 0). Previo y durante el ensayo los animales fueron mantenidos a pastoreo sobre una pradera natural fertilizada constituida principalmente por ballica (Lolium spp.), trébol blanco

\footnotetext{
Deposel® Young's Animal Health Ltd

2 Vital E-300®, Schering-Plough Animal Health
}

(Trifolium spp.), pasto ovillo (Dactylis glomerata) y pasto dulce (Holcus lanatus), cuyo contenido de Se fue de $0,04 \mu \mathrm{g} / \mathrm{g}$ base materia seca, determinado en muestras de forraje obtenidas previamente. El mantenimiento de los animales así como los procedimientos y obtención de muestras fueron supervisados por un Médico Veterinario conforme a los protocolos de manejo sanitario y productivo del predio.

\section{OBTENCIÓN DE LAS MUESTRAS}

De cada vaquilla se obtuvo una muestra de sangre sin aditivo y otra con heparina por venopunción coccígea previo a la suplementación con selenio y $\alpha$-tocoferol, previo a la revacunación, (día 0) y luego a los 30, 45 y 90 días de ensayo. Los plasmas heparinizados y sueros obtenidos fueron guardados a $-20^{\circ} \mathrm{C}$ hasta su análisis.

\section{EVALUACIÓN DEL BALANCE METABÓLICO DE SELENIO Y A-TOCOFEROL}

En las muestras de sangre heparinizada se determinó la concentración de $\mathrm{Hb}$ mediante el método de la cianometahemoglobina y se preparó un hemolizado para medir la actividad sanguínea de GPx (E.C. 1.11.1.9), según una técnica cinética compuesta NADPH-dependiente a $37^{\circ} \mathrm{C}$, usando un reactivo comercial ${ }^{3}$ en condiciones similares a las utilizadas por Leyán y col (2006). La actividad de GPx fue expresada en $\mathrm{U} / \mathrm{g} \mathrm{Hb}$. Un valor inferior a $60 \mathrm{U} / \mathrm{g} \mathrm{Hb}$ se interpretó como una carencia de selenio y sobre los 130 $\mathrm{U} / \mathrm{g}$ Hb se consideró como un balance adecuado de selenio (Ceballos y Wittwer 1996). La concentración de $\alpha$-tocoferol en plasma se determinó mediante cromatografía líquida de alto rendimiento en fase reversa en un cromatógrafo P E Nelson, acoplado a un detector UV y equipado con una columna C18 Kromasil® (150 x 4,6 mm y 4,5 $\mu \mathrm{m})$, según una adaptación a la técnica indicada por Chihuailaf y col (2008). La concentración de colesterol plasma se midió mediante el método cinético colorimétrico usando un reactivo comercial ${ }^{4}$. Se calculó la razón de masa de las concentraciones plasmáticas de $\alpha$-tocoferol/colesterol calculando el cociente de la concentración plasmática de $\alpha$-tocoferol y la concentración plasmática de colesterol (Le Blanc y col 2002).

\section{INMUNIZACIÓN CON LA VACUNA RB51}

Todos los animales fueron inmunizados con una dosis completa $\left(1-3,4 \times 10^{10}\right)$ de la vacuna Brucella abortus cepa $\mathrm{RB} 51^{5}$, mediante una inyección s.c. por delante de la

\footnotetext{
Ransel®. Laboratorios Randox

4 Cholesterol®, Human

5 Professional Biological Company, Denver, Co, USA
} 
escápula, a los tres meses possuplementación con selenio y $\alpha$-tocoferol.

\section{PREPARACIÓN ANTIGÉNICA DEL EXTRACTO TOTAL DE RB51}

La preparación antigénica de Brucella abortus utilizada en este trabajo se obtuvo de un cultivo de la cepa RB51, de acuerdo con lo descrito por Leyán y col (2006). Brevemente, Brucella abortus cepa RB51 se cultivó en caldo $\mathrm{TSB}^{6}$ a $37 \mathrm{C}^{\mathrm{o}}$ bajo agitación continua por cinco días hasta obtener un cultivo saturado con bacterias. Se centrifugó a 12.000 r.p.m. por 10 minutos y se eliminó el sobrenadante. El pellet de bacterias se suspendió en agua destilada en un volumen equivalente a $10 \%$ del volumen original del caldo saturado y se incubó bajo agitación continua en baño María a $65 \mathrm{C}^{\circ}$ por 30 minutos. Luego se le agregó $\operatorname{SDS}^{7}$ para alcanzar una concentración final de $0,5 \%$ y se incubó en un baño hirviendo por 10 minutos. La preparación se centrifugó nuevamente y el sobrenadante se utilizó como antígeno. La concentración de proteína se determinó por el método de Bradford ${ }^{8}$.

\section{EVALUACIÓN DE LA RESPUESTA INMUNE A LA VACUNA RB51}

La respuesta humoral se determinó por el método de ELISA indirecto en muestras de suero (1:2000), previamente estandarizado. Brevemente, la placa de poliestireno se sensibilizó con $0,5 \mu \mathrm{g}$ de proteína del extracto total de RB51 por pocillo y fue incubada con el suero a $4{ }^{\circ} \mathrm{C}$ durante 18 horas. Luego se utilizó como agente bloqueante leche descremada al $5 \%$ en buffer PBS T-20 incubándose a $37{ }^{\circ} \mathrm{C}$ durante una hora. Para detectar los anticuerpos específicos se utilizaron anticuerpos anti $\mathrm{IgG}$ de bovino unido a peroxidasa ${ }^{9}$. La reacción fue revelada con orto-fenilendiamina clorhidrato ${ }^{10}$ y peróxido de hidrógeno como sustrato a $450 \mathrm{~nm}$ de acuerdo con el protocolo sugerido por el fabricante. La concentración de anticuerpos se expresó en unidades de densidad óptica. El valor del punto de corte fue calculado considerando tres desviaciones estándar del promedio de la densidad óptica del control negativo. La especificidad de la reacción se determinó utilizando suero de animales no inmunizados con la vacuna y la sensibilidad se estimó en razón al número de animales vacunados que resultaron positivos.

La respuesta inmune celular se evaluó a los 90 días posvacunación mediante una prueba de intradermorreacción, previamente estandarizada para su uso en la tabla

\footnotetext{
6 Sigma, St. Louis, MO

7 Sigma, St. Louis, MO

8 Bio-Rad, Hercules CA

9 Capel, ICN Pharmaceuticals, Inc. USA

10 Sigma, USA (P8287)
}

del cuello, aplicando el extracto de B. abortus cepa RB51 antes descrito, en dosis de $32 \mu \mathrm{g}$ de proteína. El grosor de la piel fue medido con un cutímetro de resorte previo a la aplicación del extracto y luego a las 72 horas posaplicación comercial en los mismos animales. Como control del antígeno se aplicó la prueba intradérmica a vaquillas no vacunadas con la vacuna RB51.

\section{ANÁLISIS ESTADÍSTICO}

Los datos se expresaron en medias y error estándar. La normalidad y homocedasticidad de los datos fue determinada mediante la prueba de Shapiro-Wilk y la prueba de Bartlett, respectivamente. La significancia de las diferencias en las concentraciones de anticuerpos y su magnitud de cambio se evaluó mediante un análisis de varianza de medidas repetidas. La respuesta inmune celular se analizó mediante una prueba $t$ de muestras pareadas. Las diferencias en las concentraciones de $\alpha$-tocoferol y la actividad de GPx se analizaron mediante la prueba de Kruskal-Wallis usando el programa Statistix $8.0^{11}$. Se consideró significativo cuando el valor de $\mathrm{P}$ fue menor a 0,05 .

\section{RESULTADOS}

El promedio de la actividad de GPx eritrocitaria en los grupos Se, T, Se-T, y C previo a la suplementación, fue similar entre grupos $(\mathrm{P}>0,05)$. Los valores correspondieron a $69 \pm 5,8 \mathrm{U} / \mathrm{g} \mathrm{Hb}, 75 \pm 12 \mathrm{U} / \mathrm{g} \mathrm{Hb}, 81 \pm 12,2 \mathrm{U} / \mathrm{g} \mathrm{Hb}$ y $81 \pm 5,7 \mathrm{U} / \mathrm{g} \mathrm{Hb}$, respectivamente. La suplementación con selenio empleando $1 \mathrm{mg} / \mathrm{kg}$ de selenato de bario en los grupos Se-T y Se, provocó un incremento paulatino en la actividad de GPx eritrocitaria, estableciéndose diferencias $(\mathrm{P}<0,05)$ que se mantuvieron hasta el final del ensayo respecto de los grupos no suplementados con $\mathrm{Se}(\mathrm{C}$ y T)

La concentración plasmática de $\alpha$-tocoferol previo a la suplementación fue similar entre grupos $(\mathrm{P}=0,704)$ y los valores correspondieron a $4,8 \pm 0,5 \mu \mathrm{g} / \mathrm{mL}$ y $4,3 \pm$ $0,3 \mu \mathrm{g} / \mathrm{mL}$ en los grupos Se-T y Se, y de 5,0 $\pm 0,2 \mu \mathrm{g} /$ $\mathrm{mL}$ y $4,9 \pm 0,5 \mu \mathrm{g} / \mathrm{mL}$, en los grupos $\mathrm{T}$ y $\mathrm{C}$, respectivamente. La concentración plasmática de colesterol no fue diferente entre grupos $(\mathrm{P}=0,1633)$. Los valores de la razón concentración plasmática de $\alpha$-tocoferol/colesterol correspondieron a $4,2 \pm 0,3 ; 4,6 \pm 0,3 ; 3,8 \pm 0,2 ; 4,2 \pm 0,3$ para los grupos C, T, Se y Se-T, respectivamente, y fueron similares entre sí $(\mathrm{P}=0,270)$.

Al inicio del ensayo (día 0 ), los valores de la actividad sanguínea de GPx revelaron que los grupos Se y Se-T presentaban un balance adecuado de selenio y eran superiores $(\mathrm{P}<0,05)$ a los valores mostrados por los grupos no suplementados con selenio (C y T). La concentración de $\alpha$-tocoferol fue mayor en los grupos suplementados $(\mathrm{P}<0,05)$, que en el grupo control (cuadro 1). De igual

11 Analytical Sofware, USA 
Cuadro 1. Concentración plasmática de $\alpha$-tocoferol y actividad sanguínea de GPx en vaquillas a pastoreo suplementadas solo con selenio (Se), solo con $\alpha$-tocoferol (T), con selenio y $\alpha$-tocoferol (Se-T) y no suplementadas (C), durante el periodo de estudio.

Concentration of plasma of $\alpha$-tocopherol and blood activity of GPx in grazing heifers, supplemented only with selenium (Se), only with $\alpha$-tocopherol (T), with selenium and $\alpha$-tocopherol (Se-T) and unsupplemented (C) during the period of study.

\begin{tabular}{|c|c|c|c|c|c|c|c|c|}
\hline \multirow{2}{*}{$\begin{array}{l}\text { Días post } \\
\text { vacunación }\end{array}$} & \multicolumn{4}{|c|}{$\alpha$-tocoferol $(\mu \mathrm{g} / \mathrm{mL})$} & \multicolumn{4}{|c|}{ GPx (U/g Hb) } \\
\hline & $\mathrm{Se}$ & $\mathrm{T}$ & $\mathrm{Se}-\mathrm{T}$ & $\mathrm{C}$ & $\mathrm{Se}$ & $\mathrm{T}$ & Se-T & $\mathrm{C}$ \\
\hline 0 & ${ }^{\mathrm{b}} 5,4$ & b5,7 & ${ }^{\mathrm{c}} 6,5$ & $\mathrm{a} 4,5$ & $\mathrm{~b}_{120}$ & a78 & $\mathrm{b}_{142}$ & a 84 \\
\hline 28 & 4,8 & 5,5 & 5,3 & 4,7 & 135 & 90 & 170 & 103 \\
\hline 45 & 5,6 & 5,9 & 6,5 & 5,0 & 144 & 97 & 168 & 102 \\
\hline 90 & 6,8 & 7,1 & 7,8 & 6,7 & 151 & 105 & 165 & 104 \\
\hline Promedio & ab 5,7 & ${ }^{\mathrm{a}} 6,1$ & ${ }^{\mathrm{a}} 6,4$ & ${ }^{b} 5,2$ & ${ }^{b} 138$ & a93 & ${ }^{\mathrm{b}} 161$ & a98 \\
\hline Error estándar & 0,2 & 0,2 & 0,3 & 0,2 & 3,5 & 3,6 & 14,2 & 3,1 \\
\hline
\end{tabular}

(Letras diferentes en la fila indica $\mathrm{P}<0,05$ ).

manera, la relación $\alpha$-tocoferol/colesterol fue mayor en los grupos suplementados $(\mathrm{P}=0,0113)$ que en el grupo $\mathrm{C}$.

Inmediatamente previo a la administración de la vacuna RB51 (día 0), los grupos experimentales presentaron diferentes concentraciones séricas de anticuerpos $(\mathrm{P}=0,0085)$. Posteriormente, se desarrolló una curva de producción de anticuerpos en la que se registró un incremento en la concentración de anticuerpos a los 15 días posinmunización ( $\mathrm{P}$ $<0,0001$ ) en los cuatro grupos experimentales (figura 1). No obstante, la magnitud del incremento en la concentración sérica de anticuerpos en este periodo fue diferente entre grupos $(\mathrm{P}=0,0387)$ siendo menor en el grupo $\mathrm{T}$ $(\mathrm{P}<0,05)$. Posteriormente, todos los grupos tendieron a presentar menores concentraciones de anticuerpos al día 30 posinmunización. A los 45 días posinmunización, la media de la concentración de anticuerpos de los grupos

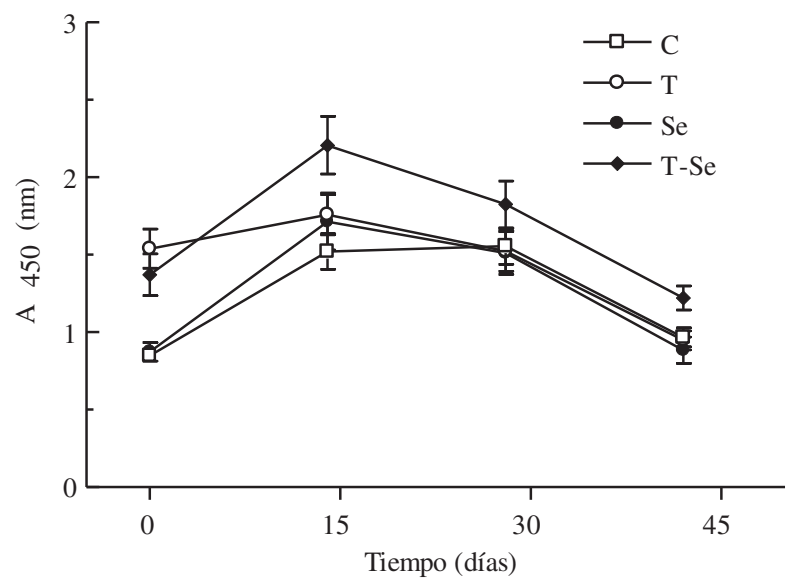

Figura 1. Cinética de la producción de anticuerpos (promedio \pm E.E.) contra proteína total de RB51, en vaquillas en pastoreo suplementadas solo con selenio (Se), solo con $\alpha$-tocoferol (T), selenio y $\alpha$-tocoferol (Se-T) y no suplementadas (C).

Kinetics of antibody production (mean \pm EE) against total protein RB51in heifers grazing supplemented with only selenium (Se), only $\alpha$-tocopherol (T), selenium and $\alpha$-tocopherol (Se-T) and unsupplemented $(\mathrm{C})$. experimentales fue menor que en el día $14(\mathrm{P}<0,001)$, sin registrarse diferencias en la magnitud de su descenso.

La prueba de intradermorreacción realizada 90 días posinmunización demostró que la respuesta inmune celular fue menor en el grupo T $(\mathrm{P}<0,05)$ y, además, se registró una tendencia a ser menor en los grupos suplementados respecto del grupo C (figura 2). Las pruebas de intradermorreacción realizadas con extracto de RB51 en vaquillas no vacunadas contra brucelosis fueron negativas.

\section{DISCUSIÓN}

De acuerdo con el promedio de la actividad sanguínea de GPx registrada en los diferentes grupos previo a la suplementación con selenato de bario, las vaquillas presentaban un balance metabólico de selenio considerado bajo marginal (Ceballos y Wittwer 1996). En animales mantenidos solo a pastoreo, el déficit de selenio es causado por el bajo contenido de este mineral en la pradera y puede agudizarse cuando la disponibilidad de forraje es inadecuada.

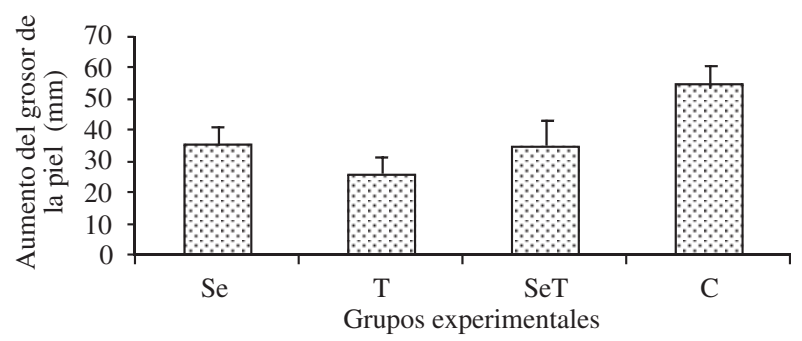

Figura 2. Aumento de grosor de la piel (promedio \pm E.E.) posterior a una intradermorreacción realizada a los 90 días posvacunación en vaquillas suplementadas con selenio (Se), $\alpha$-tocoferol ( $\mathrm{T}$ ), selenio y $\alpha$-tocoferol (Se-T) y no suplementadas (C), $(* \mathrm{P}<0,05$ respecto del control).

Increased skin thickness (mean $\pm \mathrm{SE}$ ) after an intradermal reaction performed at 90 days post vaccination in heifers supplemented with selenium (Se), $\alpha$-tocopherol (T ), selenium and $\alpha$-tocopherol (Se-T) and unsupplemented $(\mathrm{C}),(* \mathrm{P}<0.05$ relative to the control). 
En el inicio de este estudio, el balance metabólico de selenio fue diferente en los animales de los grupos Se y $\mathrm{Se}-\mathrm{T}$ respecto de los animales de los grupos $\mathrm{C}$ y $\mathrm{T}$, corroborando que la suplementación con selenato de bario provoca un incremento en la actividad de GPx; sin embargo, estos valores son inferiores a los informados por Leyán y col (2006) para animales suplementados con selenato de bario $(450 \mathrm{U} / \mathrm{g} \mathrm{Hb})$. En el transcurso de este estudio, los animales del grupo $\mathrm{C}$ y $\mathrm{T}$ presentaron un balance de selenio considerado marginal (Ceballos y Wittwer 1996) confirmando los antecedentes que señalaban que el predio presentaba forrajes con bajo contenido de selenio.

La suplementación con $\alpha$-tocoferol en los animales de los grupos $\mathrm{T}$ y Se-T estableció diferencias $(\mathrm{P}<0,05)$ en la concentración plasmática de $\alpha$-tocoferol respecto del grupo $\mathrm{C}$; no obstante estas diferencias no se evidenciaron frente al grupo Se. Las concentraciones plasmáticas de $\alpha$-tocoferol que indiquen un adecuado balance metabólico de este nutriente en vaquillas en pastoreo no se encuentran establecidas aún. Si bien, la concentración plasmática de $\alpha$-tocoferol puede ser utilizada como un estimador de este estatus metabólico, la exactitud es mayor si se corrige según la concentración plasmática de lipoproteínas proponiéndose la razón de masa $\alpha$-tocoferol/colesterol con este fin (Le Blanc y col 2002). Como ocurre con los valores plasmáticos de $\alpha$-tocoferol, tampoco se encuentran definidos los valores de referencia para la razón de masa en vaquillas preencaste; sin embargo, se acepta que valores superiores a dos en vacas lecheras indican un adecuado balance metabólico de $\alpha$-tocoferol (Baldi 2005). En general, durante el estudio se observó un incremento en la concentración de $\alpha$-tocoferol y en la razón de masa $\alpha$-tocoferol/colesterol en todos los grupos lo que es concordante con el tipo de alimentación de los animales, ya que en el periodo en que se realizó este estudio (primavera-verano) la calidad del forraje verde disponible favorece un buen aporte de vitamina $\mathrm{E}$ en animales mantenidos en pastoreo.

Se ha reportado que la carencia de selenio afecta negativamente la respuesta inmune humoral (Spears y Weiss 2008). Estudios realizados en terneros estabulados y mantenidos con un aporte marginal de selenio $(0,03-0,05$ $\mathrm{mg} / \mathrm{kg}$ alimento) tuvieron una menor respuesta inmune humoral que terneros suplementados con selenio al ser desafiados con una dosis intratraqueal de $P$. haemolytica (Stabel y col 1989), (actualmente Mannhemia haemolytica, Ewers y col 2004), o con una dosis intranasal del virus IBR (Reffett y col 1988). Los mecanismos por lo cuales el selenio influencia la respuesta inmune está dada por un efecto sobre los fagocitos y la activación, proliferación y diferenciación de linfocitos (Hoffman 2007). Por otra parte, existe evidencia que la estrecha asociación entre el Se y el $\alpha$-tocoferol puede modular la respuesta inmune (Finch y Turner 1996, Baldi 2005).

En este ensayo, la concentración sérica inicial de anticuerpos antiproteínas de Brucella fue diferente entre los grupos. En este sentido, la posibilidad de infección previa en los animales experimentales fue descartada por ser predio libre de brucelosis y la ausencia de evidencias clínicas de la enfermedad en el rebaño, por lo que el efecto residual de la primera dosis de vacuna RB51 aplicada como parte del manejo sanitario predial es el factor más plausible que explica esta observación.

Los resultados del presente estudio, en que se demuestra que la respuesta humoral a la vacuna RB51 se incrementó en todos los grupos experimentales con diferencias entre ellos, difieren con lo informado por otros autores que usaron distintos modelos experimentales. $\mathrm{Al}$ respecto, Nemec y col (1990), en un ensayo desarrollado en vaquillas suplementadas oralmente en dosis única con selenio elemental (6 g) o $\alpha$-tocoferol (1400 UI/d) o una combinación de ambas, no encontraron diferencias en la producción de anticuerpos IgG1, IgG2 o IgM entre los grupos suplementados después de ser desafiados con una dosis estándar de $3 \times 10^{10}$ UFC de B. abortus cepa 19. En otro estudio, en que se utilizaron novillos estabulados y suplementados parenteralmente con selenio o $\alpha$-tocoferol o una combinación de ambos y desafiados posteriormente con P. haemolytica ( $M$. haemolytica) se observó que la mayor respuesta de anticuerpos IgG se registró en el grupo suplementado solo con selenio y en el grupo suplementado con selenio y $\alpha$-tocoferol (Droke y Loerch 1989). Por otra parte, en un diseño diferente, vacas de 6-7 meses de gestación fueron suplementadas parenteralmente con selenio ( $0,1 \mathrm{mg}$ de selenio/kg p.v.) o con $\alpha$-tocoferol (8 UI/ kg p.v.) o una combinación de ambos y redosificados 42 días después siendo mayor la generación de anticuerpos en el grupo suplementado solo con selenio y un poco menor en el grupo suplementado con la combinación de selenio y $\alpha$-tocoferol frente a una vacuna multivalente contra E. coli (Panousis y col 2001). Es conocido el hecho que distintos tipos de antígeno generen variaciones en la respuesta humoral. En este sentido Brucella abortus induce principalmente una respuesta inmune celular Th1 (Rivers y col 2006) y una muy baja producción de anticuerpos.

Es necesario destacar que en este estudio, el grupo mantenido sin suplementación también generó una respuesta inmune humoral de igual magnitud a los grupos suplementados con selenio y $\alpha$-tocoferol. Estos resultados podrían indicar, probablemente, que los animales experimentales mantenidos en pastoreo no requerían de un aporte adicional de selenio o $\alpha$-tocoferol para generar una mayor respuesta inmune humoral, a diferencia de lo mostrado en los estudios desarrollados en animales en estabulación. De acuerdo con ello, se podría sugerir que desde la perspectiva de la inmunocompetencia, y contrario a lo señalado por la NRC (2001) y a lo planteado por Ceballos y Wittwer (1996) respecto del selenio, los animales de este estudio se encontraban en un adecuado balance del nutriente.

La vitamina $\mathrm{E}$ tiene un efecto positivo sobre la inmunidad humoral según ha sido descrito en ganado de carne (Rivera y col 2002), en ganado de leche al inicio de la lactancia (Megliá y col 2001) y en recién nacidos 
(Pekmezci y Cakiroglu 2009). No obstante, en este estudio la magnitud del incremento en la respuesta inmune fue significativamente menor en el grupo suplementado solo con $\alpha$-tocoferol. Valores de referencia para la concentración plasmática de $\alpha$-tocoferol en vaquillas no han sido definidos y solo se ha indicado que para vacas preparto el valor no debiera ser inferior a $3,0 \mu \mathrm{g} / \mathrm{mL}$ (NRC 2001). En conformidad con los resultados obtenidos se podría interpretar que, en relación con los valores de la razón de masa $\alpha$-tocoferol/colesterol, no era necesario un suministro adicional para mejorar la inmunocompetencia en los animales en estudio de tal modo que el $\alpha$-tocoferol provisto por la suplementación parenteral fue suficiente para provocar un efecto paradójico. Efectos adversos relacionados con la suplementación con altas dosis de $\alpha$-tocoferol en vacas lecheras durante el periodo seco han sido vinculados a una alta incidencia de mastitis clínica y subclínica durante la lactancia temprana (Bouwstra y col 2010). En el presente ensayo, las dosis empleadas de $\alpha$-tocoferol fueron semejantes a las dosis usadas por Bouwstra y col (2010) y se corresponden con aquellas informadas con un efecto positivo sobre la función leucocitaria (Politis y col 2004) y el estatus antioxidante hepático (Bouwstra y col 2008).

De acuerdo con lo anterior, es posible que para este nutriente sea necesario considerar relevante su relación o proporción respecto del selenio metabólicamente activo y no solo evaluar aisladamente su concentración en plasma o su razón de masa. En otras palabras, la respuesta orgánica frente a una suplementación estaría influenciada por el balance metabólico del otro nutriente. Ello podría constituir una explicación frente al hecho que en este estudio el grupo suplementado con selenio y $\alpha$-tocoferol no manifestara una disminución en la magnitud de la respuesta inmune humoral. Este planteamiento debe ser abordado en estudios posteriores. A los 45 días posinmunización, la media de la concentración de anticuerpos de los grupos experimentales fue menor al día 14 ( $\mathrm{P}<0,001)$, sin registrarse diferencias en la magnitud de su descenso.

Al analizar la respuesta inmune celular, estudios desarrollados en ratas han evidenciado que una carencia de selenoproteínas suprime la proliferación de células T (Shrimali y col 2008), disminuye la capacidad de migración de los macrófagos (Carlson y col 2010) y deprime la actividad fagocítica (Moriguchi y col 1990). También ha sido informado que la suplementación con $\alpha$-tocoferol favorece la habilidad bactericida de los neutrófilos y aumenta la función de los macrófagos en vacas (Hogan y col 1990, Hogan y col 1992, Politis y col 1995). Aunque en este ensayo, los grupos suplementados presentaron una tendencia a tener menores respuestas en la inmunidad celular, la administración parenteral de $\alpha$-tocoferol la redujo significativamente. La tendencia a la disminución de la respuesta inmune celular en el grupo suplementado solo con selenio es concordante con lo informado por Leyán y col (2005), quienes, en similares condiciones experimentales, encontraron una disminución significativa en la respuesta inmune celular en animales que presentaban una actividad sanguínea de GPx de $450 \mathrm{U} / \mathrm{g} \mathrm{Hb}$ al momento de la prueba de intradermorreacción. Es necesario precisar que en el presente estudio los valores de la actividad de GPx son inferiores a los informados por estos autores. Por otra parte, en condiciones experimentales diferentes, animales estabulados y considerados carentes de selenio que fueron suplementados con selenio a valores de GPx de $295 \mathrm{U} / \mathrm{g} \mathrm{Hb}$ presentaron una tendencia al aumento de la respuesta celular. Estos resultados están sugiriendo que la respuesta inmune celular es afectada negativamente a un determinado umbral del balance de selenio y que la suplementación con este mineral no sería recomendable cuando la actividad sanguínea de GPx supera valores de $150 \mathrm{U} / \mathrm{g} \mathrm{Hb}$ en animales en pastoreo. Si bien, Leyán y col (2005) y Leyán y col (2006) demostraron el efecto del selenio sobre la respuesta inmune celular mediante intradermorreacción, no es posible comparar la magnitud de la respuesta (grosor de la piel) entre ellos ni con el presente estudio, ya que esta depende del estatus de selenio de los animales en estudio y de las condiciones de alimentación en estabulación o pastoreo. Además del balance de selenio en los animales, otra variable importante de considerar en relación con la respuesta inmune celular es la asociación selenio y $\alpha$-tocoferol. En este sentido, del presente estudio es posible concluir que tanto la respuesta inmune celular como la humoral puede ser modulada negativamente por la concentración plasmática de $\alpha$-tocoferol al momento de la inmunización.

\section{REFERENCIAS}

Baldi A. 2005. Vitamin E in dairy cows. Livest Prod Sci 98, 117-122. Bouwstra RJ, RMA Goselink, P Dobbelaar, M Nielen, JR Newbold, $\mathrm{T}$ van Werven. 2008. The relationship between oxidative damage and vitamin $\mathrm{E}$ concentration in blood, milk, and liver tissue from vitamin E supplemented and nonsupplemented periparturient heifers. J Dairy Sci 91, 977-987.

Bouwstra RJ, M Nielen, JA Stegeman, P Dobbelaar, JR Newbold, EHJM Jansen, T van Werven. 2010. Vitamin E supplementation during the dry period in dairy cattle. Part I: Adverse effect on incidence of mastitis postpartum in a double-blind randomized field trial. $J$ Dairy Sci 93, 5684-5695.

Carlson BA, M Yoo, RK Shrimali, R Irons, VN Gladishev, DL Hatfield, JM Park. 2010. Role of selenium-containing proteins in T cell and macrophage function. Proc Nutr Soc 69, 300-310.

Ceballos A, F Wittwer. 1996. Metabolismo del selenio en rumiantes. Arch Med Vet 28, 5-18.

Ceballos A, S Espíndola, D Uslar, J Neumann, E Quiroz, R Chihuailaf, F Wittwer. 2013. Concentración de selenio en leche de estanques de rebaños lecheros del sur de Chile y su relación con indicadores productivos Arch Med Vet 45, 33-39

Chihuailaf R, P Contreras, F Wittwer. 2002. Patogénesis del estrés oxidativo: Consecuencias y evaluación en salud animal. Vet Méx 33, 265-283.

Chihuailaf RH, CS González, F Wittwer, PA Contreras. 2008. Plasma retinol concentration in grazing heifers: First data obtained from a dairy herd in the south of Chile. Arch Med Vet 40, 65-68.

Droke EA, SC Loerch. 1989. Effects of parenteral selenium and vitamin $\mathrm{E}$ on performance, health and humoral immune response of steers new to the feedlot environment. J Anim Sci 67, 1350-1359. 
Ewers C, A Lübke-Becker, LH Wieler. 2004 Mannheimia haemolytica and the pathogenesis of enzootic bronchopneumonia. Berl Munch Tierarztl 117, 97-115.

Finch JM, RJ Turner. 1996. Effects of selenium and vitamin E on the immune responses of domestic animals. Res Vet Sci 60, 97-106.

Hoffman PR. 2007. Mechanisms by which selenium influences immune responses. Arch Immunol Ther Exp 55, 1-9.

Hogan JS, KL Smith, WP Weiss, DA Todhunter, WL Schockey. 1990. Relationships Among Vitamin E, Selenium, and Bovine Blood Neutrophils. J Dairy Sci 73, 2372-237.

Hogan JS, WP Weiss, DA Todhunter, KL Smith, PS Schoenberger. 1992. Bovine Neutrophil Responses to Parenteral Vitamin E. J Dairy Sci 75, 399-405.

Le Blanc SJ, TF Duffield, KE Leslie, KG Bateman, J TenHang, JS Walton, WH Johnson. 2002. The effect of prepartum injection of vitamin E on health in transition dairy cows. J Dairy Sci 85, 1416-1426.

Leyán V, D Pesutic, G Schurig, F Wittwer, PA Contreras, J Kruze. 2005. Suplementación con selenio en vaquillas: Efecto sobre la respuesta inmune a las vacunas Brucella abortus cepa RB51 y toxoide tetánico. Arch Med Vet 37, 105-110.

Leyán V, F Wittwer, PA Contreras, G Schurig. 2006. Efecto de una dieta con bajo aporte de selenio sobre la respuesta inmune a la vacuna Brucella abortus Cepa RB51 en vacas lecheras. Arch Med Vet $38,129-135$

López-Alonso M, M Miranda, J Hernández, C Castillo, JL Benedito. 1997. Glutatión peroxidasa en las patologías asociadas a deficiencias de selenio en rumiantes. Arch Med Vet 29, 171-179.

Megliá GE, A Johanninson, L Peterson, K Persson Waller. 2001. Changes in some blood micronutrients, leukocytes and neutrophil expression of adhesion molecules in periparturient dairy cows. Acta Vet Scand 42,139-150.

Moriguchi S, N Kobayashi, Y Kishino. 1990. High dietary intakes of vitamin E and cellular immune functions in rats. J Nutr 120, 1096-1102.

Nemec M, M Hidroglu, K Nielsen, J Prolux. 1990. Effect of vitamin $\mathrm{E}$ and selenium supplementation on some immune parameters following vaccination against brucellosis in cattle. J Anim Sci 68, 4303-4309.

NRC, National Research Council. 2001. Nutrient requirements of dairy cattle. $7^{\text {th }}$ ed. National Academy Press, Washington, DC, USA.

Panousis N, N Roubies, H Karatzias, S Frydas, A Papasteriadis.2001. Effect of selenium and vitamin $\mathrm{E}$ on antibody production by dairy cows vaccinated against Escherichia coli. Vet Rec 149, 643-646.

Pekmezci, D, D Cakiroglu. 2009. Investigation of immunmodulatory effects of levamisole and vitamin E on immunity and some blood parameters in newborn Jersey calves. Vet Res Comm 33, 711-721.

Politis I, M Hidiroglou, TR Batra, JA Gilmore, RC Gorewit, H Scherf. 1995. Effects of vitamin $\mathrm{E}$ on immune function of dairy cows. $\mathrm{Am}$ $J$ Vet Res 56, 179-184.

Politis I, I Bizelis, A Tsiaras, A Baldi. 2004. Effect of vitamin E supplementation on neutrophil function, milk composition and plasmin activity in dairy cows in a commercial herd. J Dairy Res $71,273-278$.

Stabel JR, JW Spears, TT Brown, J Brake. 1989. Selenium effects on glutathione peroxidase and the immune response of stressed calves challenged with Pasteurella hemolytica. J Anim Sci 67, 557-564.

Reffett JK, JW Spears, TT Brown. 1988. Effect of dietary selenium on the primary and secondary response in calves challenged with infectious bovine rhinotracheitis virus. J Nutr 118, 229-235.

Rivera JD, GC Duff, ML Galyean, DA Walker, GA Nunnery. 2002. Effects of supplemental vitamin E on performance, health, and humoral immune response of beef cattle. J Anim Sci 80, 933-941.

Rivers R, E Andrews, A González-Smith, G Donoso, A Oñate. 2006. Brucella abortus: inmunidad, vacunas y estrategias de prevención basadas en ácidos nucleicos. Arch Med Vet 38, 7-18.

Shrimali RK, RD Irons, BA Carlson, Y Sano, VN Gladyshev, JM Park, DL Hatfield. 2008. Selenoproteinas mediate T cell immunity through an antioxidant mechanism. J Biol Chem 283, 20181-20185.

Spears JW, WP Weiss. 2008. Role of antioxidants and trace elements in health and immunity of transition dairy cows. Vet $J 176,70-76$. 